\title{
Comment on Man: That Near-sighted Creature
}

\section{INTRODUCTION}

R oughly one million years BP Homo erectus, thanks to an extraordinary development of its brain's capabilities, learned to manufacture relatively elaborate tools and to use fire, whereas its predecessor Homo habilis had used only rough stones - much as certain otters, a few birds, and some apes, still do.

Probably what happened was that some random cosmic or other event caused unique changes in the genetic code of Homo erectus that specifically enhanced its brainpower. In any case some genetic mutations turned out to be most eventful: they made it possible first for Homo erectus, then for its immediate successor Homo neanderthalensis, and lastly for today's Homo sapiens, to enrich his knowledge and capabilities one bit after another, the experience of each generation being transmitted through language to the next, with a powerful cumulative effect that is still ongoing.

After the end of the last glacial era, about 10 thousand years BP and only some 400 human generations ago, Man changed again - from a hunter-gatherer to a farmer-breeder, thanks to the revolutionary invention of agriculture.

\section{The Last Two Centuries}

During the following millennia, Man's progress was steady but slow — until about 200 years ago (just 8 normal generations before us), when a new crucial turning-point was reached. The process of learning and transmitting new knowledge accelerated dramatically: the dawn of the industrial revolution, in fact, marked the advent of great scientific discoveries, of ever-more-daring technical inventions (the steam-engine first and foremost), and of extraordinary progress in science and medicine. The amount of accumulated knowledge was by then so great that every new 'conquest' became the springboard for still more advanced conquests, and the consequences were soon obvious: in vast areas of the planet Man's living conditions improved greatly, infant mortality shrank drastically, many diseases were overcome or effectively harnessed, and human life-expectancy was extended more and more until it almost doubled.

At the same time, however, something else began to take place: namely an unrestricted and uncontrolled proliferation of the human species - soon amounting to a veritable infestation of the planet — and with it came an irrepressible increase and range of accompanying injuries to the environment. Unfortunately, the very mechanism of gradual acquisition of knowledge and experience that characterized the cultural development of Man (as opposed to one that would allow the sudden acquisition of knowledge in bulk), prevented him from seeing the negative medium- and long-term consequences of his discoveries and inventions on the environment, on Man himself, and on planetary life. Thus Man never even thought of implementing the necessary safeguards, being fully occupied and complacently contented with the resulting short-term benefits: ever-improving living conditions, and consolidation of his supremacy on Earth.

In many ways, Man behaved for a long time as an unrepentant opener of Pandora's boxes, with no clue as to how he could put back into the boxes what had spilled from them - or worse still, not even realizing that such a need existed.

So, Man was for long unable to foresee the long-term consequences of his own uncontrolled multiplication; of the polluting techniques he invented to produce and use energy and to manufacture products; of the depletion of the forest patrimony; and of the squandering of non-renewable fossil energy and minerals.

Only after two disastrous centuries of such improvident conduct, when the damage has become massive and difficult to remove, does the awareness begin to surface at all widely that Mankind is heading towards disaster - to avoid which strong remedies must be found urgently. It is just as if Man had suffered during his entire evolution from a congenital mental near-sightedness that only gave him a short-range, personally selfish vision.

Paradoxically, a subspecies that somewhat immodestly called itself 'sapiens sapiens' was chronically caught unawares by the effects of its own actions and omissions.* As a result, such problems as global overpopulation, and environmental pollution and degradation, in reality fully predictable from the beginning if only one had thought of them ${ }^{\dagger}$, and not too difficult to ward off by acting early, now appear suddenly, almost as unexpected bad news or surprises.

\section{SEVERAL EXAMPLES OF NEAR-SIGHTEDNESS}

Many examples of human near-sightedness can be listed. The first, and no doubt most lethal because it acts as a multiplier for all the others, was the blind exploitation of the impressive progress of medical science which drastically reduced mortality and stretched life-expectancy without the slightest concern - at any time, even at present - for the absolute need to lower birth-rates correspondingly, so as to keep the global superecocomplex in sustainable balance.

\footnotetext{
* Inspiring the late Sir Julian Huxley, first Director-General of UNESCO — and we ourselves since in affectionate emulation - to refer to the human species as 'Homo misnamed sapiens'. For Sir Julian had been our predecessor as a biological Fellow of New College, Oxford, and, much later, the ever-helpful Scienfific Patron of our First International Conference on Environmental Future. — Ed.

$\dagger$ As we did with due consideration in many instances in our International Conferences on Environmental Future, of which the first took place a year before 'Stockholm' and was supposedly a useful input thereto as we carried each chapter of the proceedings, after editing it, the short distance to the Palais des Nations, Geneva, where preparations were being made for what is yet often referred to as the beginning of the environmental movement. - Ed.
} 
A second example of near-sightedness was the invention of the combustion engine and motorized vehicles running on it: both apparently clever and valuable innovations which, however, have the serious drawback of using a non-renewable and highly polluting energy source; the resulting environmental damages, while tolerable and digestible on a small scale, have by now become massive and unbearable because of the explosive multiplication of motor vehicles, now (1995) exceeding 550 millions world-wide, while the total number of combustion engines of all types is estimated at 700 millions.

Few even suspected in the past that, in the long run, the polluting emissions and unburned particles discharged from the vehicles' exhaust-pipes would cause intolerable air pollution, erosion of monuments and buildings, acid rains that can prove lethal to plants and animal wildlife, respiratory ailments and tumours, and even measurable alterations in the composition of gases in the atmosphere - potentially increasing the 'greenhouse effect' and threatening catastrophic climatic changes.

Again, no one in the past apparently realized that, whereas the fuelling of a few automobiles involved only trifling environmental problems, the vast multiplication of circulating vehicles would require an everincreasing number of environmentally damaging oil-wells for drilling; of dangerous pipelines and tankers for transportation; of polluting refineries; and of hazardous and cumbersome tank-trucks for distribution.

A third example of human near-sightedness was the use of mineral oil and its derivatives as an energy-source also for manufacturing, heating, and power generation: surely in many ways a more convenient source than wood or coal, and yet, like coal, a finite and non-renewable source in human-scale times, but most of all gravely polluting. Until very recently nobody seemed to care about those drawbacks or about the fact that proven oil reserves world-wide are sufficient for no more than a few dozen years at present consumption-rates - and anyhow oil consumption in the world continues at an unreduced rate, or indeed on a grander scale than ever!

A fourth example of Man's near-sightedness was his adoption of nuclear energy for power generation. The commonsense rule of selecting fool-proof technologies, if they exist at all, in such hazardous applications, was never followed; nor was the problem ever addressed of disposing of, or neutralizing, large quantities of spent nuclear rods, which would still remain dangerously radioactive for thousands of years.

The consequences were, on one hand, the well-known accidents of Three Mile Island and of Chernobyl (whose full effects are still only partially known), not to speak of similar cases that have never been made public, and of those to be expected in the future, as there are now more than 400 nuclear, fission-based powerplants on the planet - just as unsafe or only slightly less so than the two just named.

On the other hand, the radioactive waste generated by those power-plants, that nobody knows how to get rid of, is now stored in several hundred 'temporary' sites: just so many 'floating mines' for future generations, true monuments to human recklessness and foolishness.

Only now is the need dawning on us to design 'intrinsically safe' fission power-plants (if this should be possible); but the problem of radioactive waste remains unsolved - including the disposal of radioactive materials resulting from the power-plants themselves when dismantled at the end of their life. Of these there are an estimated quantity which is greater than that produced in the form of spent rods in the 40 years of operation.

A fifth example of Mankind's near-sightedness is its widespread utilization of plastics (which are also mainly oil derivatives) to manufacture articles of all types and apparently quite useful, ranging from shopping bags to car bumpers. No one seems to know what to do about the growing mountains of urban and industrial refuse made of non-biodegradable, indestructible, and non-recyclable, plastics - except for accumulating such materials in garbage-dumps in the hope that future generations will find a better solution, or burning them in incinerators that produce dioxin and other harmful gases.*

But even the ubiquitous, unpretentious paper (our sixth chosen example of human near-sightedness) has grown to represent a serious environmental problem. More and more paper is being used - even when no real need exists, and even when it would be easy and often more convenient to do without it, as in the excessive use of packing materials and of many throw-away articles (diapers, etc.), not to mention the frequent use of multiple layers of packaging and binding, etc. Whole forests have been and still are being destroyed to produce paper and cardboard, with irreparable environmental degradation plus air, water, and soil, pollution. Yet only recently, and rather timidly, the use of recycled paper, manufactured from second-hand paper, has been found acceptable, if still hesitatingly, and not many countries and cities have yet introduced a working system of separate garbage collection for paper, preparatory to recycling.

The list of examples of human near-sightedness (often sheer foolishness) could be much longer, but, having cited some random examples, let us now ask the following question.

\footnotetext{
* Here we think of an occasion a few years ago when we were telephoned by a celebrated specialist in ocean pollution about his fear that the ocean floor would in time become 'lined with' discarded plastic, and would we consider a paper about it? But as his subsequent submission did not deal with this topic, we concluded that he had come to think of his environmental fear, for once, as having been exaggerated. - Ed.
} 


\section{ARE THERE No REMEDIES?}

The answer is yes, even though they are not painless and presuppose a generalized awareness that, should Mankind go on along the present course, not only the well-being, but the survival itself of the coming generations - and perhaps even of life on Earth - would be placed in serious jeopardy.

The remedies consist mainly in a vigorous and effective effort to bring back the totality of Mankind to a level that would be durably sustainable by the planetary superecocomplex; to develop clean means of locomotion and renewable, non-polluting energy sources; to convert city wastes into valuable resources to be almost wholly recycled and reutilized; and to force manufacturers to take back and recycle all used industrial articles.

But, in addition, if modern Man is really to deserve the self-granted designation of sapiens sapiens, the lethal human near-sightedness must be corrected once and for all: for example by introducing regulations to the effect that in future each new gadget, invention, or innovation (past, present, and future), before being approved and released for practical use, must undergo a strict and impartial investigation — by an independent Authority with world-wide jurisdiction - as to possible medium- or long-term environmental effects also affecting future generations; and obviously, such innovations must be banned if their effects prove unacceptable, unless adequate and compulsory countermeasures can be found whereafter further testing should be mandatory.

GiACOMO DE SABATA Via Bigli 4, I-20121 Milano, Italy.

UPDATED ANNOUNCEMENT: Change of Publisher and Editor

\section{Change of Publisher and Editor}

From the first issue of 1996, Environmental Conservation will be published by Cambridge University Press. Correspondence concerning subscriptions, permissions, and other matters related to the publication and distribution of the Journal, should be directed as follows:

In the UK and rest of the world except North America and Mexico:

Journals Department, Cambridge University Press, The Edinburgh Building, Shaftesbury Road, Cambridge CB2 2RU, UK,

Tel. +441223312393

Fax +44 1223315052 .

In USA, Canada, and Mexico:

Journals Department, Cambridge University Press, 40 West 20th Street,

New York, NY 10011-4211, USA

Tel. +12129243900

Fax +1 2126913239 .

Submission of manuscripts should be to: Dr N.V.C. PoLUNIN

Editor of Environmental Conservation

Department of Marine Sciences \& Coastal Management

Ridley Building

University of Newcastle

Newcastle upon Tyne WEI 7RU

England, UK

from whom Instructions for Authors* and further details may be obtained:

$$
\begin{aligned}
& \text { Tel. + } 44 \text { 191-222-6675 } \\
& \text { Fax + } 44 \text { 191-222-7891 } \\
& \text { Telex 53654 (UNINEW G) } \\
& \text { e-mail=n. polunin@ newcastle. ac. uk }
\end{aligned}
$$

* which should be followed in the interests of time and other economy. 